\title{
Untersuchung des spektralen Einkoppelverhaltens von Lichtwellenleitersensoren
}

\author{
Harald Beyer, Heinz Pursch, Andreas Schumacher
}

\section{Einleitung}

In Schaltanlagen des Niederspannungsnetzes können Störlichtbögen zu erheblichen Schäden fuihren. Die Ursache der Lichtbögen ist vielfältig und reicht von Überschlägen an Isolationsstrecken uiber Blitzeinschläge in das Netz bis zu Kurzschlüssen, die bei Wartungs- oder Montagearbeiten verursacht werden können. Die Wirkungen von Störlichtbögen sind erheblich und können die Zerstörung der Schaltanlage zur Folge haben. Die Klöckner-Moeller GmbH hat ein Störlichtbogenschutzsystem (ARCON) entwickelt, das in die Schaltanlagen integriert wird. Da das Auftreten der Störlichtbögen nicht vorhersehbar ist, wird die gesamte Schaltanlage von Sensoren auf der Basis von Lichtwellenleitern überwacht. Für diesen Einsatzzweck sind die Sensoren so konzipiert, dass das Licht des Bogens nicht über die Stirnfläche des Lichtleiters, sondern seitlich über den Mantel in den Kern eingekoppelt wird. Die Leuchterscheinungen jedes sich bildenden Lichtbogens können von dem Sensor erfasst und an einen Detektor weitergeleitet werden, dessen Signal die Schutzmaßnahmen einleitet. Dadurch kann der Strom noch während der ersten Halbwelle abgeschaltet und der Schaden begrenzt werden.

Um das System zu optimieren, sind Informationen über das spektrale Einkoppel- und Übertragungsverhalten von Lichtwellenleitersensoren vom ultravioletten bis in den infraroten Wellenlängenbereich notwendig. Es ist wichtig zu wissen, wieviel Strahlungsleistung in Watt pro Wellenlänge für das Nachweissystem zur Verfügung steht. Die Messergebnisse sind deshalb mit einem Strahlungsnormal zu kalibrieren.

Hauptproblem der Untersuchung ist die äußerst geringe Lichtmenge, die bei seitlicher Bestrahlung in den Lichtwellenleiter gelangt. Für hohe Intensitäten, wie sie kurzzeitig bei Störlichtbögen auftreten, entstehen durchaus gut nachweisbare Lichtimpulse im Lichtleiter. Für ein kontinuierlich strahlendes Strahlungsnormal bei eher moderaten Strahlungsleistungen erfordert der Nachweis einen erheblichen Detektionsaufwand.

Der physikalische Prozess für die seitliche Einkopplung war zu Beginn der Untersuchungen unklar. Die Einkopplung ist nur über einen Prozess denkbar, der für Licht, das im Kern des Lichtleiters geführt wird, einen Verlustprozess darstellt. Das Licht kann den Lichtleiter nur verlassen, wenn der Auftreffwinkel auf die Grenzfläche zum Mantel den Winkel der Totalreflexion unterschreitet. Das bedeutet aber, dass Licht, das vom Mantel in den Kern gelangt, nicht im Kern geführt werden kann, sondern ihn wieder verlässt. In Betracht kommen nur Oberflächen-, Streu- und Biegungsverluste.
Dass an starken Biegungen der Faser Licht gut eingekoppelt werden kann, ist bekannt und experimentell bestätigt. Dieser Effekt ist aber für die hier vorgesehenen Untersuchungen störend und sollte vermieden werden. Störungen der Grenzschicht zwischen Mantel und Kern wären ein weiterer möglicher Grund für die Einkopplung. Jedoch wird bereits bei der Herstellung von Lichtleitern darauf geachtet, dass dieser Effekt minimal ist. Streuprozesse (Rayleighstreuung) an diesen Störungen sind ebenfalls denkbar. Dann sollte die Streuintensität indirekt proportional zur vierten Potenz der Wellenlänge sein, was durch die Untersuchungsergebnisse zu verifizieren wäre.

\section{Experimentelles}

\subsection{Messaufbau}

Die Messung aller Spektren erfolgte mit einem 0,75 mSpektrographen der Firma Acton unter Verwendung eines Gitters mit 150 Strichen /mm. Die Aufzeichnung der Spektren wurde mit einer intensivierten CCD-Kamera mit einer Auflösung von 376 x 1024 Pixeln vorgenommen. Der Kamera vorgeschaltet ist ein Lichtverstärker, der auftreffende Photonen in Elektronen umwandelt, die dann vervielfacht werden. Anschließend werden sie wieder in Licht umgewandelt, das die CCD-Kamera registriert. Die Verstärkung beträgt etwa $10^{6}$. Wegen des Lichtverstärkers erstreckt sich der Empfindlichkeitsbereich der Kamera von $200-870$ nm. Das Gesamtspektrum wurde aus drei einzelnen Teilbildern zusammengesetzt.

Um das Signal des Lichtleiters im Spektrographen maximal zu nutzen, muss die Apertur des Lichtleiters an die des Spektrographen angepasst werden. Dies geschieht durch eine zusätzlich angebrachte Spiegelanordnung (Abb. 1).

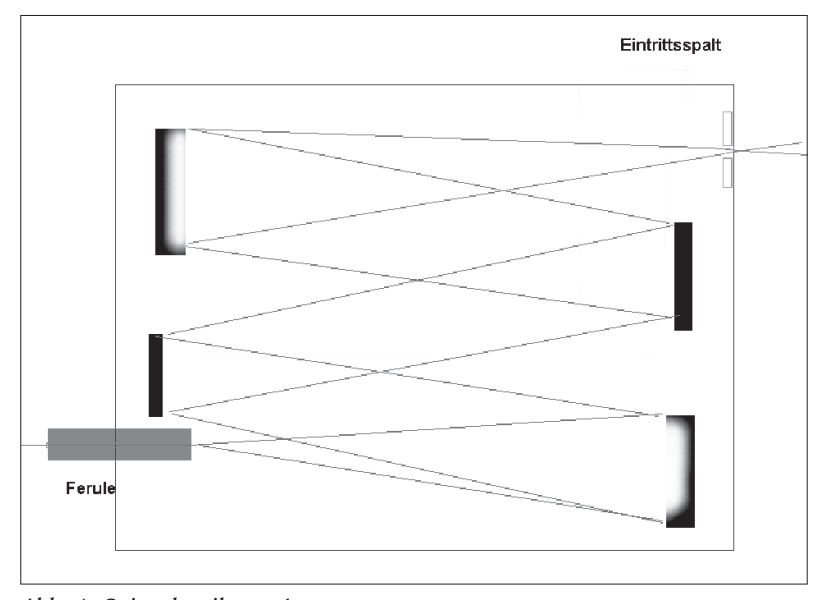

Abb. 1: Spiegeloptik zur Aperturanpassung 
Das spektral aufgelöste Bild der Lichtwellenleitersensoren beleuchtet nur einen Teil der CCD-Matrix. Die nicht verwendeten Teile der Matrix wurden ausgeblendet, da sie nicht zum Nutzsignal beitragen, sondern nur den Dunkelstromanteil vergrößern. Der Dunkelstrom wurde bei abgedunkelter Messanordnung ermittelt und von jedem Spektrum subtrahiert. Jedes Spektrum besteht aus $970 \mathrm{x}$ 33 Pixeln. Die x-Richtung der Kamera entspricht der Wellenlänge (mit Eichspektren kalibriert), und in y-Richtung wird die Intensität addiert. Das Spektrum ist von Pixel 24 bis Pixel 950 in x-Richtung nutzbar. Am langwelligen Rand des Spektrums wird die Kamera durch eine Blende nicht mehr beleuchtet. Dieser Teil der CCD-Matrix wird zur Dunkelstromkontrolle verwendet. Zur Minimierung des Dunkelstroms wird die CCD-Kamera mit einem Peletierkühler gekühlt. Die Wärme wird durch Kühlwasser abgeführt. Schwankungen der Kühlwassertemperatur haben somit Auswirkung auf den Dunkelstrom. Diese Dunkelstromschwankungen haben bei geringen Intensitäten und langen Messzeiten Einfluss auf das Messergebnis und müssen eliminiert werden. Dazu wird der abgeschattete Teil der CCD-Matrix verwendet. Die Werte der Pixel 955970 wurden gemittelt und vom Spektrum subtrahiert. Bei vollständiger Abdunklung erhält man auf diese Weise eine gute Nulllinie. Die Abbildung 2 zeigt Bilder, die mit der CCD-Kamera bei $470 \mathrm{~nm}$ und $630 \mathrm{~nm}$ Mittelwellenlänge aufgenommen wurden. Als Lichtquelle wurde ein kontinuierlicher Strahler (Halogenlampe) verwendet. Um einen deutlichen Effekt zu erzielen, wurde hier eine hohe Intensität verwendet, was sich in einer Überstrahlung des Bildes äußert. Der Bereich des Spaltbildes und der zur Dunkelstromsubtraktion verwendete abgeschattete Bereich sind deutlich zu erkennen.

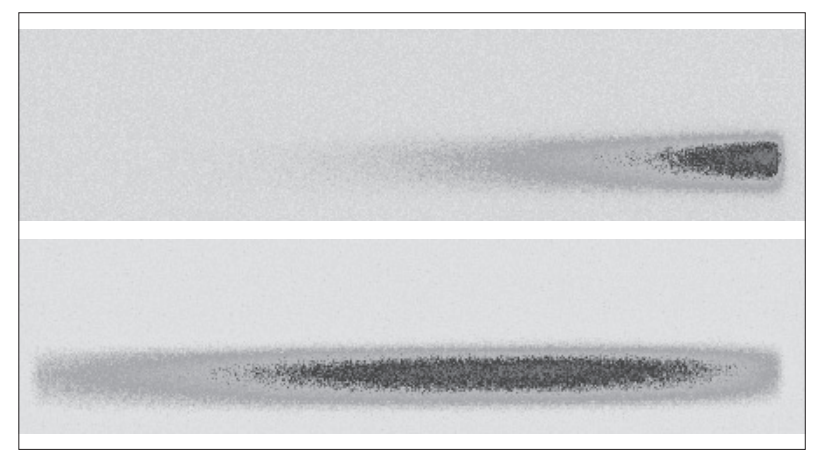

Abb. 2: CCD-Kamerabilder bei $470 \mathrm{~nm}$ und $630 \mathrm{~nm}$ Mittelwellenlänge

\subsection{Durchführung der Messung}

Wegen der geringen Intensitäten wird für alle Spektren eine relativ lange Belichtungszeit von 150 ms verwendet. Jede Messung wird 40 mal wiederholt und das Ergebnis akkumuliert. Die Einkopplung erfolgte an vier unterschiedlichen Positionen des Lichtleiters.

Zur Vermeidung unerwünschter Einkopplung an Biegungen wurden die Fasern auf einem optischen Tisch von $2 \mathrm{~m}$ x 1,6 m mit großen Radien ausgelegt und mit schwarzem, lichtdichten Tuch abgedeckt. Um Streulicht zu vermeiden, erfolgten die Messungen in einem abgedunkelten Raum, so dass die Fasern nur an den vorgesehenen Messpositionen von der Ulbrichtkugel (Strahlungsnormal) bestrahlt wurden. Besonders diffizil war die Abdeckung

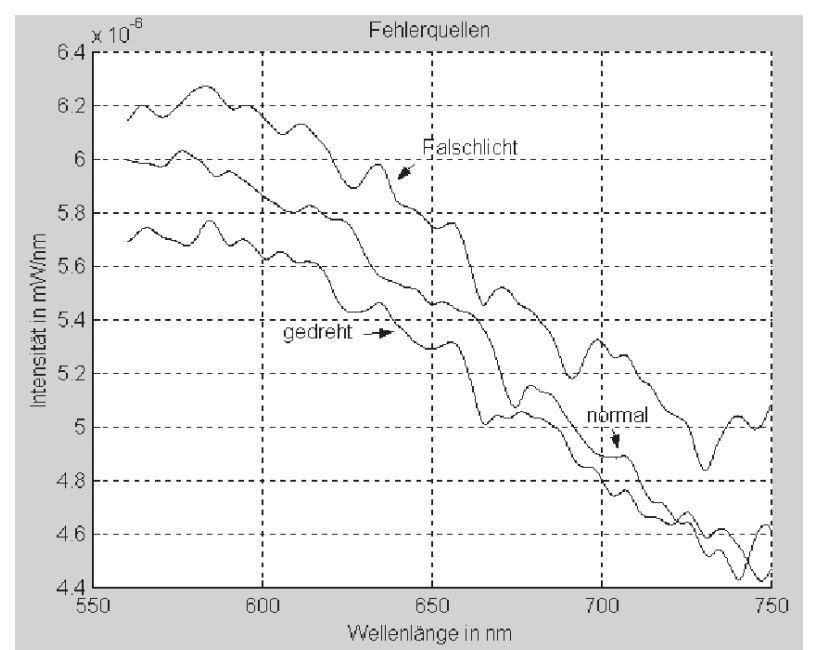

Abb. 3: Einfluß möglicher Fehler auf das gemessene Spektrum

für die dünnen Fasern im Bereich der Öffnung der Ulbrichtkugel, da sie leicht zu Durchbiegungen neigen. Abbildung 3 verdeutlicht den Einfluss möglicher Fehler beim Einkoppeln in die Faser. Nimmt man die mittlere Kurve als Normalkurve, dann zeigt im Vergleich dazu die obere Kurve die Wirkung von Falschlicht. Hier wurde der Lichtleiter in der Nähe der Messposition an der Ulbrichtkugel nicht abgedeckt. Dadurch wird mehr Licht in die Faser übertragen. Das wird zum einen durch eine größere bestrahlte Faserlänge verursacht. Zum anderen wird durch die Faserkrümmung, die sich bei der Zufuihrung zur Kugel nicht ganz vermeiden lässt, mehr Licht eingekoppelt. Die untere Kurve zeigt das Messergebnis für den selben Lichtleiter bei Bestrahlung der Faser von einer anderen Seite (Drehung der Faser in der Halterung). Bei dem veränderten Resultat ist zu beachten, dass nicht nur ein anderer Oberflächenabschnitt bestrahlt wird, sondern auch durch die Bewegung des LWL's andere Biegeradien auftreten.

Die Kalibrierdaten der Ulbrichtkugel sind in Abbildung 4 dargestellt. Daraus wurden durch eine Spline-Interpolation für die drei verwendeten Spektrometereinstellungen Datensätze erzeugt, die den Wellenlängen der Messwerte entsprechen. Damit liegt für die verwendeten Spektralbereiche zu jedem Messwertpaar (Wellenlänge, Intensität) ein entsprechendes Wertepaar mit Kalibrierdaten vor.

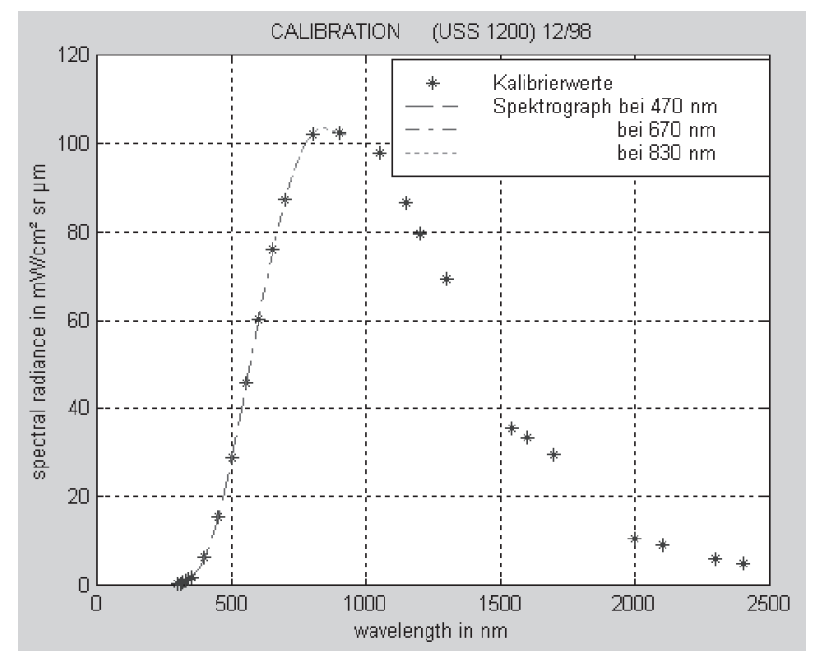

Abb. 4: Kalibrierdaten der Ulbrichtkugel und Spline-Interpolation für die drei verwendeten Spektrometereinstellungen 


\section{Auswertung der Messungen}

Für das von der Kamera aufgezeichnete Signal sind die Einkopplung in die Faser und die Lichtleitung in der Faser, aber auch die optischen Übertragungseigenschaften der Aperturanpassung, des Spektrographen und der Kamera einschließlich des Verstärkers maßgeblich. Wie bereits einführend erwähnt, ist die äußerst geringe Lichtmenge, die bei side-on-Bestrahlung in den Lichtwellenleiter gelangt, das Hauptproblem der Messungen. Abbildung 5 verdeutlicht die vorliegenden Verhältnisse. Die obere Abbildung zeigt die end-on-Einkopplung der Strahlung der Ulbrichtkugel (Bestrahlung der Öffnung Lichtleiterferule) und die untere Abbildung die side-onEinkopplung (Einstrahlung uiber die Mantelfläche). Bei gleichen Belichtungszeiten ist das end-on-Signal nur mit

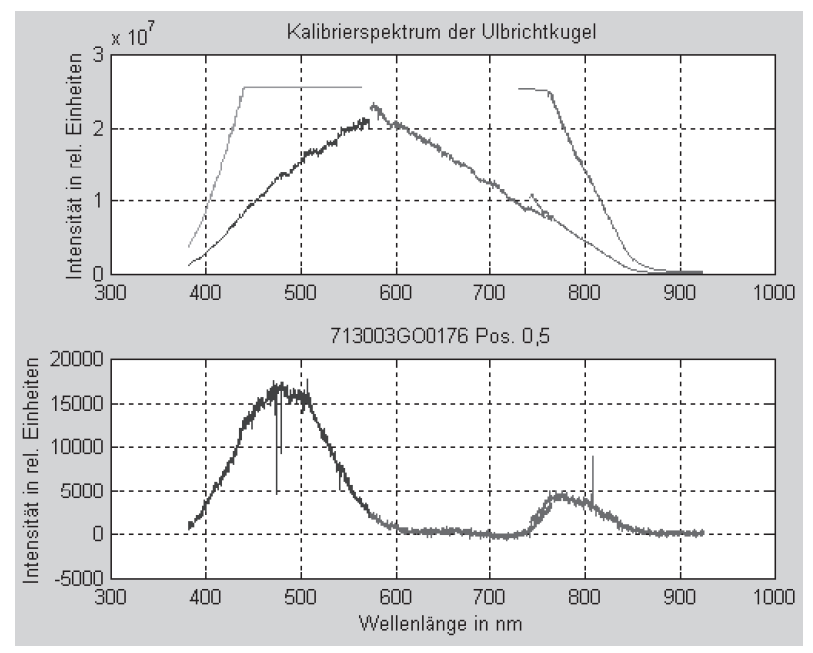

Abb. 5: Unterschiede zwischen end-on- und side-on-Einkopplung (unten)

einem Filter messbar. Der Unterschied in der eingekoppelten Intensität liegt je nach Fasertyp zwischen $10^{4}$ und $10^{5}$. In der Konsequenz erhält man einen verhältnismäßig großen Rauschanteil an den gemessenen Spektren. Lange Messzeiten begünstigen das Auftreten von Spikes, die beim Durchgang von Höhenstrahlung durch den Detektor verursacht werden. Die negativen Spikes entstehen bei der Subtraktion des Untergrundsignals. Die Messsignale werden deshalb mit einem kubischen Glättungspline geglättet.

Um erste Übersichtsdarstellungen über die Einkopplung zu erhalten, wurde das Messsignal der side-on-Einkopplung mit der Kalibrierkurve der Ulbrichtkugel multipliziert und bezüglich der end-on-Einkopplung dieser Faser normiert. Daraus lassen sich noch keine Absolutwerte ableiten, da die Parameter der Fasern wie bestrahlte Fläche und Querschnitt nicht berücksichtigt sind. Trotzdem sind Vergleiche des spektralen Verhaltens der unterschiedlichen Fasertypen möglich.

Die Messergebnisse für einen Lichtwellenleitersensor sind in Abbildung 6 dargestellt (der Kalibrierfaktor ist in der Darstellung bereits berücksichtigt) und zeigen, dass es keine Korrelation zwischen der Messposition und der gemessenen Intensität gibt.

Für dieses Verhalten gibt es zwei Ursachen: Erstens ist die Dämpfung innerhalb der Faser außerordentlich gering, da es sich um Quarzglasfasern handelt. Die Dämpfungsfaktoren sind für den von uns untersuchten

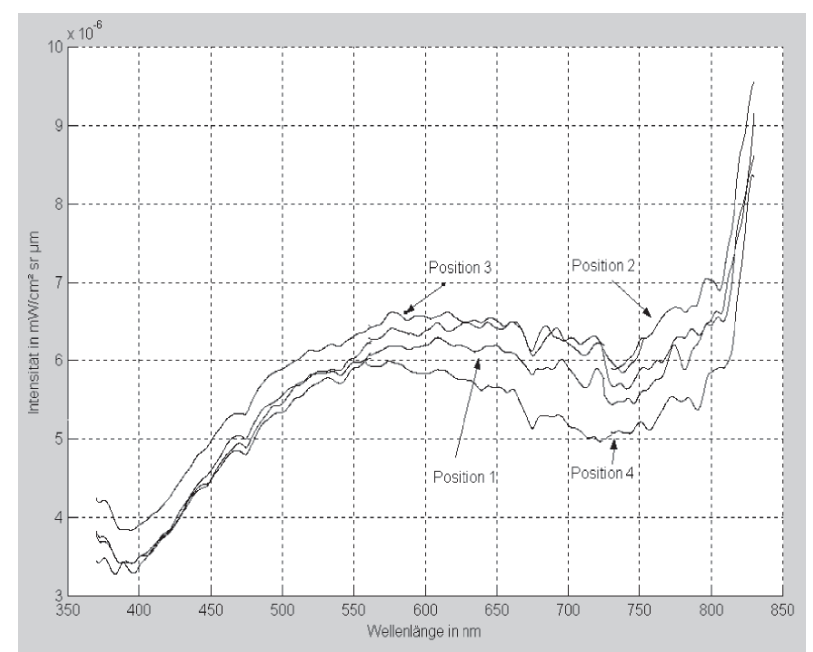

Abb. 6: Absolutwerte der an verschiedenen Positionen über Mantel eingekoppelten Strahlung in Abhängigkeit von der Wellenlänge

Wellenlängenbereich in der Größenordnung von nur 2 $55 \mathrm{~dB} / \mathrm{km}$. Die Charakterisierung der LWL durch einen von der Wellenlänge abhängigen Dämpfungsfaktor erscheint deshalb als nicht sinnvoll.

Zweitens ist die Einkopplung in die Faser von den lokalen Eigenschaften der Mantel/Kern-Grenzfläche abhängig. Die tatsächlich an einer bestimmten Position eingekoppelte Intensität ist mit einer zufälligen Komponente behaftet und führt zu Intensitätsschwankungen an den verschiedenen Messpositionen.

Da auch keine Zunahme der Intensität bei kleinen Wellenlängen zu beobachten ist, können Streuprozesse für den Einkoppelprozess ausgeschlossen werden.

Bei der Ermittlung von Absolutwerten der über den Mantel eingekoppelten Strahlung muss berücksichtigt werden, dass die jeweilige Faser nicht nur Messobjekt ist, sondern auch als Teil des optischen Systems in Erscheinung tritt. Dadurch mögliche Fehler können sich aus der Güte der polierten Endfläche der Faser, der numerischen Apertur und dem Faserdurchmesser ergeben. Bei der weiteren Auswertung zur Kalibrierung der Einkopplung uiber die Mantelfläche werden nun der Durchmesser der Faser und die bestrahlte Fläche berüicksichtigt. Die bestrahlte Fläche ergibt sich aus dem Produkt von Länge der Faser vor der Öffnung der Ulbrichtkugel $(\varnothing 110 \mathrm{~mm})$ und dem Kerndurchmesser der jeweiligen Faser.

Die Fasern befinden sich bei den Messungen in guter Näherung auf der Mantelfläche der Ulbrichtkugel, so dass die Faser Licht aus einem Raumwinkel von 2 p aufnehmen kann. Die spektral eingekoppelte Leistung in Watt je Nanometer ergibt sich dann aus der folgenden Beziehung:

\section{$\mathrm{P} \lambda=\underline{\text { Messsignal }} \mathrm{I}_{\text {-side-on }} \stackrel{ }{*}$ Kalibrierkurve der Ulbrichtkugel Messsignal $\mathrm{I}_{\text {end-on }}{ }^{*}$ Fläche * $2 \pi$}

In allen Spektren tritt bei einer Wellenlänge von $800 \mathrm{~nm}$ ein starker Anstieg der eingekoppelten Leistung auf. Obwohl der Anstieg nahe dem Ende des Beobachtungsintervalls liegt und die Empfindlichkeit der Kamera in diesem Bereich geringer wird, kann der spektrale Verlauf bis $850 \mathrm{~nm}$ verfolgt werden. Dennoch könnten die Daten mit einem größeren Fehler behaftet sein als solche, die im mittleren Teil des Spektrums gemessen wurden. 


\section{Zusammenfassung}

Dieses Messverfahren wurde auf unterschiedliche Lichtwellenleitersensoren der Klöckner-Moeller GmbH angewendet.

Das spektrale Verhalten der Lichtwellenleitersensoren bei seitlicher Bestrahlung konnte im Wellenlängenbereich von $380 \mathrm{~nm}$ bis $850 \mathrm{~nm}$ vermessen und der Intensitätsverlauf absolut kalibriert werden. Die Einkopplung wird offenbar hauptsächlich durch die Eigenschaften des Übergangs Mantel/Kern bestimmt. Die beobachteten Intensitätsschwankungen weisen auf lokale Änderungen der Einkoppeleigenschaften hin.

\section{Danksagung}

Wir danken Herrn Prof. Dr. G. Fußmann und Herrn Dr. W. Bohmeyer für die Unterstuitzung mit Geräten und Erfahrungen bei der Durchführung der Untersuchungen im MPI für Plasmaphysik Berlin.

Der Firma PI Physik Instrumente, insbesondere Herrn Dr. Dr.-Ing. R. Behrendt, gilt unser Dank fuir die Bereitstellung von Infrarotdioden.

Herr G. Richter war uns mit Glasfasermesstechnik der Firma ANRITSU GmbH behilflich.

\section{Autoren}

\section{Dipl.-Phys. Harald Beyer}

Technische Fachhochschule Wildau Institut fuir Plasma- und Lasertechnik Tel. (0 33 75) 508-126

E-Mail: hjbeyer@igw.tfh-wildau.de

\section{Dr. Heinz Pursch}

Institut für Niedertemperatur-Plasmaphysik Greifswald Friedrich-Ludwig-Jahn-Str. 19

17489 Greifswald

E-Mail: pursch@inp-greifswald.de

\section{Andreas Schumacher}

Klöckner-Moeller GmbH

Hein-Moeller-Straße 7-11

53115 Bonn

E-Mail: aschumacher@moeller.net 